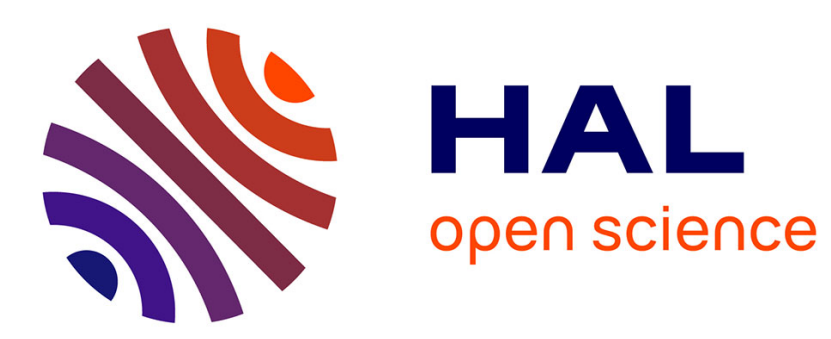

\title{
Compte-rendu. Soraya Boudia, Nathalie Jas, Gouverner un monde toxique
}

Julien Pongerard

\section{To cite this version:}

Julien Pongerard. Compte-rendu. Soraya Boudia, Nathalie Jas, Gouverner un monde toxique. Lectures, 2019, 10.4000/lectures.35475 . halshs-03332467

\section{HAL Id: halshs-03332467 \\ https://shs.hal.science/halshs-03332467}

Submitted on 16 Sep 2021

HAL is a multi-disciplinary open access archive for the deposit and dissemination of scientific research documents, whether they are published or not. The documents may come from teaching and research institutions in France or abroad, or from public or private research centers.
L'archive ouverte pluridisciplinaire HAL, est destinée au dépôt et à la diffusion de documents scientifiques de niveau recherche, publiés ou non, émanant des établissements d'enseignement et de recherche français ou étrangers, des laboratoires publics ou privés. 


\section{Soraya Boudia, Nathalie Jas, Gouverner un monde toxique}

\section{Julien Pongerard}

\section{(2) OpenEdition}

Journals

Electronic version

URL: https://journals.openedition.org/lectures/35475

DOI: $10.4000 /$ lectures.35475

ISSN: 2116-5289

\section{Publisher}

Centre Max Weber

Brought to you by Université de Strasbourg

\section{이마실 Les bibliothéques \\ de l' Université de strasbourg}

\section{Electronic reference}

Julien Pongerard, "Soraya Boudia, Nathalie Jas, Gouverner un monde toxique", Lectures [Online], Reviews, Online since 25 June 2019, connection on 16 September 2021. URL: http:// journals.openedition.org/lectures/35475 ; DOI: https://doi.org/10.4000/lectures.35475

This text was automatically generated on 16 September 2021.

(C) Lectures - Toute reproduction interdite sans autorisation explicite de la rédaction / Any replication is submitted to the authorization of the editors 


\title{
Soraya Boudia, Nathalie Jas, Gouverner un monde toxique
}

\author{
Julien Pongerard
}

1 Depuis plusieurs années, Soraya Boudia et Nathalie Jas participent au développement d'une réflexion collective, entre sociologie, histoire et science politique, sur la contamination du monde par des substances toxiques, sur ses conséquences concrètes, et sur les modes de gouvernement qui l'ont rendue - et continuent de la rendre possible. Parmi les jalons de ce programme de recherche, on peut citer deux livres qu'elles ont codirigés, Toxicants, Health and Regulation since $1945^{1}$ et Powerless Science? The Making of the Toxic World in the Twentieth Century2 ${ }^{2}$. Le petit opus recensé ici en reprend les conclusions et fournit aux autrices l'occasion de présenter, sous une forme synthétique et plus accessible au lectorat francophone que les introductions aux ouvrages sus-cités, un certain nombre de leurs idées fortes sur la gouvernance contemporaine des polluants et des pollués.

2 La description glaçante de Baotou, une ville de Mongolie-Intérieure intensément polluée, qui ouvre le livre, pose le cadre de la réflexion menée par la suite. Elle introduit la définition du monde matériellement toxique qui fait l'objet du premier chapitre, tout en attirant l'attention sur des conséquences parfois éludées de l'intoxication historique et effrénée du monde : celle-ci se concrétise dans l'intoxication de tous les écosystèmes, mais aussi des corps humains, et particulièrement ceux de populations défavorisées. Ce monde toxique est une réalité objectivable à travers le nombre et la quantité croissante de substances polluantes mises en circulation sur la planète (produits chimiques, métaux lourds, nanoparticules, perturbateurs endocriniens, etc.), et à travers « l'abyssale immensité » (p. 27) de leurs conséquences présence et persistance de ces substances dans tous les milieux; maladies et décès plus ou moins visibles, etc.

3 La démarche d'enquête socio-historique permet aux autrices de pointer le caractère construit du monde toxique. Il est le résultat d'un processus d'intoxication globalisé, accéléré après 1945 avec le développement d'une économie fondée sur les "productions industrielles et technoscientifiques» (p. 10). Il se donne à voir dans la 
croissance effrénée de la production de substances toxiques - y compris celles dont la toxicité justifie l'interdiction dans de nombreux pays, comme le plomb (p. 18) -, les cas de catastrophes polluantes qui égrènent l'actualité, ou les millions de décès annuels estimés qui leur sont liés. Ce développement économique est accompagné par un ensemble de dispositifs politiques, scientifiques et sociaux qui le rendent à la fois possible et acceptable. Ce sont ces configurations historiques, "ensembles assez cohérents de discours, de pratiques, d'instruments, d'outils, d'institutions et d'acteurs " qui définissent et organisent «ce que doit être une bonne prise en charge des substances chimiques dangereuses et leurs effets » (p. 6), que les autrices appellent des modes de gouvernement et dont elles distinguent trois modalités.

4 S. Boudia et $\mathrm{N}$. Jas décrivent en premier lieu un mode de gouvernement "par la maîtrise » qui promet, à partir de 1945, de réguler et de contrôler la toxicité et les effets néfastes des substances chimiques dont la production s'accélère. Le dispositif de gestion privilégié est celui des normes et l'outil principal de régulation est la fixation de seuils maximaux de toxicité (concentrations maximales de certaines substances dans les aliments, doses journalières admissibles, etc.). Ces seuils, négociés entre diverses parties prenantes, participent d'une «logique d'accommodement» (p. 46) entre les organismes gouvernementaux qui cherchent à limiter les effets des substances toxiques et les représentants d'industries chimiques qui veulent éviter l'interdiction de ces substances.

5 Le scandale lié à la révélation de la toxicité du DDT participe d'une « crise de toxicité » (p. 62), avec laquelle émerge dans les années 1970 un nouveau mode de gouvernement, qui n'est plus tourné vers une maîtrise de la pollution mais vers la gestion des risques qui y sont associés. Face à l'essor des contestations environnementales et de nouveaux savoirs scientifiques et militants sur la toxicité, ce mode de gouvernement "par les risques » reconnaît les dangers inhérents et inévitables du développement industriel de produits synthétiques toxiques. Dans le même temps, il installe un cadre d'évaluation et de décision fondé sur la quantification probabiliste des pollutions et de leurs dégâts. Cela favorise leur acceptabilité sociale en promouvant l'idée que le risque est connu et maitrisé, et les éventuelles conséquences néfastes compensables. Des outils de gestion comme l'analyse risque-bénéfice aident alors à construire des compromis au sein de larges panels d'intérêts concurrents, en instaurant une démarche générique d'évaluation des risques, et en établissant un niveau socialement acceptable de risque.

Ces garanties sont cependant régulièrement mises en échec, notamment à travers des épisodes historiques catastrophiques comme l'accident nucléaire de Tchernobyl, dont l'ampleur déborde le cadre de la gestion par les risques. Diverses institutions (autorités gouvernementales, instituts de recherche, entreprises...) mènent sur les sites durablement pollués des expériences de gestion, en reportant sur les populations la responsabilité de s'adapter et de mettre en place des stratégies pour vivre et rendre habitable un monde toxique. Dans ce nouveau paradigme de l'adaptabilité, l'action des gouvernants tend à se limiter à la fourniture d'informations sur les niveaux de pollution et les risques associés aux individus concernés, et à l'accompagnement du « vivre-avec » dans un monde inéluctablement empoisonné. Cette conceptualisation du gouvernement par l'adaptation, largement reprise de l'introduction à Toxicant, repose sur des bases moins étendues que les deux autres, reflétant le caractère peu développé des recherches sur le sujet. Néanmoins, S. Boudia et N. Jas en font l'emblème d'un mode de gouvernement contemporain en développement, inscrit dans le droit fil d'une 
gouvernance néolibérale et individualisante des risques, et dans une dynamique de dérégulation (p.97). Ce mode de gouvernement reporte cyniquement sur les victimes de pollution la responsabilité d'éviter leur propre empoisonnement, et, sous couvert d'un «empowerment " valorisant, ajoute aux contraintes et à la culpabilisation qui peuvent peser sur des populations vulnérables (femmes en charge des décisions familiales, populations géographiquement marginalisées, individus moins diplômés, etc.) - alors même que les populations concernées n'ont souvent pas d'autre choix que de vivre avec les pollutions, ni de responsabilité individuelle dans les pollutions. Les autrices font ainsi apparaître la possibilité d'une perpétuation de l'« art de gouverner des populations» (p. 92), jusque sur les « ruines capitalistes» où Anna Tsing pointait plutôt les possibilités de s'y soustraire dans un ouvrage récent et remarquée.

7 En évoquant un grand nombre de cas d'étude, les autrices montrent la multiplicité des enjeux qui participent à l'élaboration et l'évolution des modes de gouvernement par le contrôle, le risque et l'adaptation - une tripartition chronologique qui semble utile, heuristiquement, pour analyser différents dispositifs de gestion contemporains. Un nouveau mode émerge lorsque le précédent est disqualifié par les conséquences des pollutions qu'il n'arrive plus à contenir et par les critiques subséquentes dont il fait l'objet. Il intègre ainsi les critiques existantes et actualise l'horizon des promesses de gestion de l'intoxication du monde, en s'appuyant sur les appareils de connaissance techno-scientifiques et les dispositifs de gestion disponibles au moment de sa gestation - on pourra à cet égard utilement compléter les analyses du rôle des savoirs scientifiques dans l'élaboration d'un mode de gouvernement avec celles de Sara Aguiton ${ }^{5}$ sur les dispositifs de gouvernement des technosciences par les acteurs et intérêts techno-industriels.

Par-delà l'évolution historique, S. Boudia et $\mathrm{N}$. Jas pointent surtout avec acuité et dans une perspective critique la constance de certaines dynamiques. Les différents modes de gouvernement privilégient systématiquement la préservation du potentiel de développement économique des marchés de substances toxiques, au détriment de la protection des populations contre les pollutions. En s'appuyant sur de nombreux exemples de débats, d'acteurs et d'outils, mais aussi sur les tentatives de remises en cause régulières (et leurs succès tout relatifs dans l'avènement de "prises de conscience » souvent impuissantes), l'ouvrage met clairement en évidence le caractère politique et négocié des décisions prises quant à la gestion des substances toxiques et de leurs effets, et le caractère historique et stratégique d'une "naturalisation » (p. 91) des risques de pollution, que les différents modes de gouvernement rendent socialement acceptables.

9 Tant par sa bibliographie foisonnante que par les conceptualisations opérationnelles proposées, cet ouvrage aux conclusions sombres mais éclairantes fournit une synthèse efficace pour mieux comprendre ce que gouverner un monde toxique veut dire depuis 1945 , et propose une analyse critique du fonctionnement contemporain de la gouvernance politique, scientifique et technique qui perpétue une intoxication générale aux conséquences inégalement distribuées. 


\section{NOTES}

1. Jas Nathalie, Boudia Soraya (dir.), Toxicants, health and regulation since 1945, Londres et New York, Pickerng \& Chatto, 2013.

2. Boudia Soraya, Jas Nathalie (dir.), Powerless science? Science and politics in a toxic world, Londres et New York, Bergahn Books, 2011.

3. Ce terme est employé par Sarah Vogel pour désigner les bouleversements des années 1960 et 1970 aux États-Unis, dans Is it Safe ? BPA and the Struggle to Define the Safety of Chemicals. University of California Press, Berkeley, 2013.

4. Tsing Anna, Le champignon de la fin du monde. Sur la possibilité de vivre dans les ruines du capitalisme, Paris, La Découverte, 2017.

5. Aguiton Sara, La Démocratie Des Chimères. Gouverner La Biologie Synthétique, Lormont, Le Bord de l'eau, 2018.

\section{AUTHOR}

\section{JULIEN PONGERARD}

Doctorant en sociologie à l'UMR GESTE (Irstea/ ENGEES). Il est lauréat de l'agrégation de sciences économiques et sociales. 